\title{
PROPER MOUFANG SETS WITH ABELIAN ROOT GROUPS ARE SPECIAL
}

\author{
YOAV SEGEV
}

\section{INTRODUCTION}

Moufang sets are the Moufang buildings of rank one. They were introduced by J. Tits [T1] as a tool for studying algebraic groups of relative rank one. They are essentially equivalent to split $B N$-pairs of rank one, and as such they have been studied extensively. In some sense they are the basic 'building blocks' of all split $B N$-pairs. In the finite case, it had been a major project to classify split $B N$-pairs of rank one. This project culminated in [HKSe]. Moufang sets are also essentially equivalent to Timmesfeld's 'abstract rank one groups' (see [Ti1]). In recent years there has been a revived interest and significant progress in this area; for additional information, see the bibliography at the end of the paper.

Let us recall that a Moufang set is essentially a doubly transitive permutation group such that the point stabilizer contains a normal subgroup which is regular on the remaining points. These regular normal subgroups are called the root groups, and they are assumed to be conjugate and to generate the whole group. In DW. DS1, DS3 the notation $\mathbb{M}(U, \tau)$ is used for a Moufang set (and this notation is of course explained there). The group $U$ in this notation is isomorphic to any one of the root groups of the Moufang set.

Recall that $\mathbb{M}(U, \tau)$ is special iff $(-a) \tau=-(a \tau)$, for all $a \in U^{*}$. We say that $\mathbb{M}(U, \tau)$ is a sharply 2-transitive Moufang set if its little projective group is sharply 2 -transitive. Recall that a Moufang set is proper if it is not sharply 2-transitive. In this paper we prove:

Main Theorem. Let $\mathbb{M}(U, \tau)$ be a proper Moufang set such that $U$ is abelian. Then $\mathbb{M}(U, \tau)$ is special.

The Main Theorem is proved in section 11. It resolves a main conjecture in the area of Moufang sets. We note that although the condition that $\mathbb{M}(U, \tau)$ is 'special' seems at first somewhat technical, this condition has many implications on $\mathbb{M}(U, \tau)$ (see DW, DS1, DST]). Indeed, the condition that $\mathbb{M}(U, \tau)$ is special should eventually lead to the identification of $\mathbb{M}(U, \tau)$ as $\mathbb{M}(J)$ for some quadratic Jordan division algebra $J$. We refer the reader to [Ti1, DW, DS1] and [Ti2 for results on special Moufang sets with abelian root groups and their connection to quadratic Jordan division algebras.

Received by the editors February 19, 2008.

2000 Mathematics Subject Classification. Primary 20E42; Secondary 17C60.

Key words and phrases. Moufang set, root group.

The author was partially supported by BSF grant no. 2004-083.

(C)2009 American Mathematical Society 
For the sake of completeness we also recall that the converse of the Main Theorem should also hold:

Conjecture 1. Let $\mathbb{M}(U, \tau)$ be a special Moufang set. Then $U$ is abelian.

We refer the reader to DST and S2 for partial results on this conjecture.

Recall from [S1, section 3] the notion of a 'root subgroup' (see also DS3, section $6]$ ). Here are the main steps in the proof of the Main Theorem. The first crucial step is to show that, under the hypotheses of the Main Theorem, if $a \in U^{*}$ is such that $\mu_{a}$ is an involution and $\sim a \neq-a$ (see the notation below for (the important) $\sim a$ ), then $\left\{b \in U^{*} \mid \mu_{b}=\mu_{a}\right\} \cup\{0\}=: V_{a}$ is a root subgroup of $U$ (Theorem 7.1). Note that a priori there is no reason in the world why $V_{a}$ should be a subgroup (!). The second crucial step is to show that if we assume in addition that all the $\mu$-maps are involutions, then the theorem holds (Theorem 8.1). The third important step is to show that $\mu_{a}$ is an involution, for any $a \in U^{*}$ such that $\sim a \neq-a$ (Theorem 9.1). Finally, letting $I=\left\{a \in U^{*} \mid \mu_{a}^{2}=1\right\}$, the final crucial step is to show that $I \cup\{0\}$ is a root subgroup of $U$ (Theorem 11.5). The Main Theorem follows quickly.

Notice that a Moufang set $\mathbb{M}(U, \tau)$ such that $\mu_{x}=\mu_{y}$, for all $x, y \in U^{*}$ is a sharply 2-transitive Moufang set, because by DW, Theorem 3.1(ii)] the Hua subgroup $H$, which is, by definition, the pointwise stabilizer of 0 and $\infty$, is generated by $\left\{\mu_{x} \mu_{y}^{-1} \mid x, y \in U^{*}\right\}$, so it is trivial. In particular, for the root subgroup $V_{a}$ above, the corresponding Moufang set $\mathbb{M}\left(V_{a}, \tau\right)$ is sharply 2-transitive. Thus, along our work on the Main Theorem we have encountered situations where, working for a contradiction, we get nontrivial sharply 2-transitive root subgroups properly contained in $U$ (for example, the root subgroups $V_{a}$ or the root subgroup $I \cup\{0\}$ above), where $\mathbb{M}(U, \tau)$ is a proper Moufang set. We conjecture that this never happens:

Conjecture 2. Let $\mathbb{M}(U, \tau)$ be a proper Moufang set, and let $V \leq U$ be a root subgroup with $|V| \geq 5$. Then $\mathbb{M}(V, \tau)$ is proper.

The reason for the condition $|V| \geq 5$ in Conjecture 2 is to exclude the cases $\mathrm{PSL}_{2}(2) \cong \mathrm{S}_{3}$ (the case $\left.|V|=2\right), \operatorname{PSL}_{2}(3) \cong \mathrm{A}_{4}$ (the case $|V|=3$ ) and the case $\mathrm{Sz}(2)(|V|=4) 1 \mathrm{Sz}(2)$ is a Frobenius group of order 20). These cases occur as (little projective groups of) root subgroups of proper Moufang sets.

Call a Moufang set $\mathbb{M}(U, \tau)$ Zassenhaus if the Hua subgroup $H$ acts semiregularly on $U \backslash\{0\}$ (i.e. the only permutation fixing 3 points is the identity). It seems to us that it would be important to investigate Zassenhaus Moufang sets as they are, in some sense, minimal Moufang sets. We cannot however, at this time, formulate a conjecture on how these Moufang sets should look; so we ask:

Question 3. What is the structure of Zassenhaus Moufang sets?

A word on notation. We conclude the introduction with a word on notation. The notation in this paper follows [DS1, section 2]; see also [DST, section 1]. The notation $\mathbb{M}(U, \tau)$ is of course explained there. Recall that $\sim a:=\left(-a \tau^{-1}\right) \tau=$ $\left(-a \mu_{-x}\right) \mu_{x}$, for all $x \in U^{*}$, and that by [DS1, Prop. 3.10(3)], $\sim a=-(-a) \mu_{a}$. Notice that $\mathbb{M}(U, \tau)$ is special iff $\sim a=-a$, for all $a \in U^{*}$. Note also that $\sim a=$ $\sim b \Longleftrightarrow a=b$, for all $a, b \in U^{*}$. Throughout this paper we let

$$
V_{a}:=\left\{b \in U^{*} \mid \mu_{a}=\mu_{b}\right\} \cup\{0\}, \quad a \in U^{*} .
$$

\footnotetext{
${ }^{1}$ We thank Hendrik Van Maldeghem for pointing out this case to us.
} 
The following standard facts will be used without further reference:

$$
\mu_{-a}=\mu_{a}^{-1}=\mu_{\sim a}, \mu_{a \mu_{b}}=\mu_{-a}^{\mu_{b}}, \mu_{a}^{h}=\mu_{a h}, \quad \forall a, b \in U^{*} \text { and } h \in H .
$$

Recall also that

$$
h \in \operatorname{Aut}(U), \quad \text { for all } h \in H,
$$

and that by DS1, Proposition 3.10(5) and equation (3.3)]:

$$
\mu_{c}=\mu_{-b} \mu_{b-a} \mu_{a} \quad \text { and } \quad c=(a-b) \mu_{b}+\sim b, \text { where } c=\left(a \tau^{-1}-b \tau^{-1}\right) \tau .
$$

Main Hypothesis. Throughout this paper $\mathbb{M}(U, \tau)$ is a Moufang set where $\tau=\mu_{e}$ for some $e \in U^{*}$. Further, except in section 2, we assume that $U$ is abelian.

\section{Lemmas for General Moufang Sets}

In this section $\mathbb{M}(U, \tau)$ is an arbitrary Moufang set.

Lemma 2.1. Let $a \in U^{*}$. Then

(1) if $\sim a=a$, then $(a \tau) \cdot 2=0$;

(2) if $\sim a=a$ and $\sim-a=-a$, then $a \cdot 2=0$;

(3) if $a \mu_{a}=a$ and $(-a) \mu_{a}=-a$, then $a \cdot 2=0$;

(4) if $(-a) \mu_{a} \in\{a,-a\}$, for all $a \in U^{*}$, then $\mathbb{M}(U, \tau)$ is special.

Proof. (1): We have $a \tau=(\sim a) \tau=-(a \tau)$, so (1) holds.

(2) and (3): We first note that the hypotheses in (2) and (3) are equivalent. Indeed using [DS1, Prop. 3.10(3)] we see that $\sim a=a$ iff $(-a) \mu_{a}=-a$ and that $\sim-a=-a$ iff $-a \mu_{-a}=-a$ iff $a \mu_{a}=a$.

Assume that $\sim a=a$ and $\sim-a=-a$. By (1), with $\tau=\mu_{a}$ we get that $\left(a \mu_{a}\right) \cdot 2=$ 0 , so since $a \mu_{a}=a$, we have $a \cdot 2=0$.

(4): Assume that for some $a \in U^{*},(-a) \mu_{a} \neq a$. Then $(-a) \mu_{a}=-a$ and $a \cdot 2 \neq 0$. Thus, since $\mu_{a}^{2} \in \operatorname{Aut}(U)$, we have $a \mu_{a}^{2}=-\left[(-a) \mu_{a}^{2}\right]=a$, and it follows that $a \mu_{a}=a \mu_{-a}=a$ (because $a \mu_{-a} \neq-a$ ). By (2), $a \cdot 2=0$, a contradiction.

Hence we see that

$$
(-a) \mu_{a}=a, \text { for all } a \in U^{*} \text {. }
$$

Hence $\sim a=-(-a) \mu_{a}=-a$, for all $a \in U^{*}$, so $\mathbb{M}(U, \tau)$ is special.

Corollary 2.2. Let $\mathbb{M}(U, \tau)$ be a Moufang set. Then $\mathbb{M}(U, \tau)$ is special iff the implication

holds for all $a, b \in U^{*}$.

$$
\mu_{a}=\mu_{b} \quad \Longrightarrow \quad a= \pm b
$$

Proof. If $\mathbb{M}(U, \tau)$ is special, then the implication holds by [DS1, Prop. 4.9(4)]. Suppose the implication holds. By equation (1.2), $\mu_{(-a) \mu_{a}}=\mu_{a}$, for all $a \in U^{*}$, and hence $(-a) \mu_{a}= \pm a$. Then, by Lemma 2.1 (4), $\mathbb{M}(U, \tau)$ is special.

Lemma 2.3. Let $a \in U^{*}$. Then

(1) $\sim a=-(-a) \mu_{a}$;

(2) $\sim-a=-a \mu_{-a}$;

(3) $-\sim a=(-a) \mu_{a}$;

(4) $\sim-\sim a=a \mu_{a}$;

(5) $-\sim-a=a \mu_{-a}$;

(6) $\sim-\sim-a=(-a) \mu_{-a}$;

(7) $-\sim-\sim a=-a \mu_{a}$; 
(8) $\sim-\sim-\sim a=-a \mu_{a}^{2}$;

(9) $-\sim-\sim-a=-(-a) \mu_{-a}$;

(10) $\sim-\sim-\sim-a=a \mu_{-a}^{2}$;

(11) $-\sim-\sim-\sim a=a \mu_{a}^{2}$.

Proof. First note that $(-a) \mu_{x}=\sim\left(a \mu_{x}\right)$ and $(\sim a) \mu_{x}=-\left(a \mu_{x}\right)$, for all $x \in U^{*}$. Part (1) is [DS1, Prop. 3.10(3)], and (2), (3) are immediate from (1). Part (5) is immediate from (2). Next, by (3),

$$
\sim-\sim a=\sim(-a) \mu_{a}=\sim\left(\sim\left(a \mu_{a}\right)\right)=a \mu_{a},
$$

so (4) holds. By (5),

$$
\sim-\sim-a=\sim a \mu_{-a}=(-a) \mu_{-a},
$$

so (6) holds. Part (7) follows from (4). By (7) and (2) (with $a \mu_{a}$ in place of $a$ ),

$$
\sim-\sim-\sim a=\sim\left[-\left(a \mu_{a}\right)\right]=-\left[a \mu_{a} \mu_{-a \mu_{a}}\right]=-a \mu_{a}^{2},
$$

and (8) holds. Part (9) comes from (6), and (11) comes from (8). For (10) replace in (11) $a$ with $-a$ to get $\sim-\sim-\sim-a=-(-a) \mu_{-a}^{2}=a \mu_{-a}^{2}$, since $\mu_{-a}^{2} \in \operatorname{Aut}(U)$.

Lemma 2.4. Let $a \in U^{*}$. If $V_{a} \subseteq\{0, a,-a\}$, then $\sim a=-a$.

Proof. Notice that $-\sim a, \sim-a \in V_{a}$. Suppose that $V_{a} \subseteq\{0, a,-a\}$ and assume that $\sim a \neq-a$. Then $-\sim a=-a$, that is $\sim a=a$, and $a \cdot 2 \neq 0$. Also $\sim-a=-a$, so by Lemma 2.1](2), $a \cdot 2=0$, a contradiction.

Lemma 2.5. Let $a, b \in U^{*}$ and assume that $a \neq b$ and $\mu:=\mu_{a}=\mu_{b}$. Then

(1) $\mu_{a-b}=\mu_{a \mu^{-1}-b \mu^{-1}}$;

(2) $\mu_{-a+b}=\mu_{-\sim a+\sim b}$.

Proof. (1): Using equations (1.2) and (1.4) we get

$$
\begin{aligned}
\mu^{-1} \mu_{a \mu^{-1}-b \mu^{-1}} \mu & =\mu_{\left(b \mu^{-1}-a \mu^{-1}\right) \mu} \\
& =\mu_{-a} \mu_{a-b} \mu_{b} \\
& =\mu^{-1} \mu_{a-b} \mu
\end{aligned}
$$

so (1) holds.

(2): Replacing $a$ with $-a$ and $b$ with $-b$ in (1) we must replace $\mu^{-1}$ with $\mu$, so we get

$$
\mu_{-a+b}=\mu_{(-a) \mu-(-b) \mu} .
$$

Notice however that $(-a) \mu=(-a) \mu_{a}=-\sim a$ and similarly $-(-b) \mu=\sim b$, so $(2)$ holds.

Lemma 2.6. Let $a, b \in U^{*}$ and assume that $a \neq b$ and $\mu_{a}=\mu_{-b}$. Then $\mu_{a-b}=$ $\mu_{a \mu_{a}^{-1}-b \mu_{a}^{-1}} \mu_{a}^{2}=\mu_{a}^{2} \mu_{a \mu_{a}-b \mu_{a}}$.

Proof. We have

$$
\begin{aligned}
\mu_{a}^{-1} \mu_{a \mu_{a}^{-1}-b \mu_{a}^{-1}} \mu_{a} & =\mu_{\left(b \mu_{a}^{-1}-a \mu_{a}^{-1}\right) \mu_{a}} \\
& =\mu_{-a} \mu_{a-b} \mu_{b} \\
& =\mu_{a}^{-1} \mu_{a-b} \mu_{-a}
\end{aligned}
$$


so the first equality holds. The second equality holds because by equation (1.2) and since $\mu_{a}^{2} \in \operatorname{Aut}(U)$ we get

$$
\mu_{a \mu_{a}^{-1}-b \mu_{a}^{-1}} \mu_{a}^{2}=\mu_{a}^{2} \mu_{-a}^{2} \mu_{a \mu_{a}^{-1}-b \mu_{a}^{-1}} \mu_{a}^{2}=\mu_{a}^{2} \mu_{a \mu_{a}-b \mu_{a}} .
$$

\section{Two BASIC LEMMAS}

In this section we prove two lemmas that are some of the main tools used in this paper. We remind the reader that from now on we assume that $U$ is abelian.

Lemma 3.1. Let $a, b \in U^{*}$ with $a \neq b$ and $\mu_{a}=\mu_{b}$. Then

(1) $(a-b) \mu_{b}-(b-a) \mu_{-b}=\sim-b+a-\sim b$;

(2) $\left(a \tau^{-1}-b \tau^{-1}\right) \tau-\left((-a) \tau^{-1}-(-b) \tau^{-1}\right) \tau=a$;

(3) $(a-b) \tau-((\sim a)-(\sim b)) \tau=a \tau$.

Proof. (1): We have

$$
\left(a \tau^{-1}-b \tau^{-1}\right) \tau=\left(-b \tau^{-1}+a \tau^{-1}\right) \tau=\left((\sim b) \tau^{-1}-(\sim a) \tau^{-1}\right) \tau .
$$

Now, by equation (1.4),

$$
\left(a \tau^{-1}-b \tau^{-1}\right) \tau=(a-b) \mu_{b}+(\sim b)
$$

and

$$
\left((\sim b) \tau^{-1}-(\sim a) \tau^{-1}\right) \tau=(\sim b-\sim a) \mu_{-a}+a
$$

Furthermore

$$
\begin{aligned}
(\sim b-\sim a) \mu_{-a} & =\left(-(-b) \mu_{b}+(-a) \mu_{a}\right) \mu_{-a} \\
& =\left((-a) \mu_{a}-(-b) \mu_{a}\right) \mu_{-a} \\
& =(-a+b) \mu_{-b}+\sim-b .
\end{aligned}
$$

Comparing (3.2) and (3.3) we get

$$
(a-b) \mu_{b}+\sim b=(-a+b) \mu_{-b}+\sim-b+a,
$$

so (1) holds.

(2): By equation (3.2) we have

$$
(a-b) \mu_{b}=\left(a \tau^{-1}-b \tau^{-1}\right) \tau-\sim b,
$$

and replacing $a$ with $-a$ and $b$ with $-b$ we get

$$
(b-a) \mu_{-b}=\left((-a) \tau^{-1}-(-b) \tau^{-1}\right) \tau-\sim-b .
$$

Hence, by (1) we get

$$
\begin{aligned}
\sim-b+a-\sim b & =(a-b) \mu_{b}-(b-a) \mu_{-b} \\
& =\left[\left(a \tau^{-1}-b \tau^{-1}\right) \tau-\sim b\right]-\left[\left((-a) \tau^{-1}-(-b) \tau^{-1}\right) \tau-\sim-b\right] \\
& =\left(a \tau^{-1}-b \tau^{-1}\right) \tau-\left((-a) \tau^{-1}-(-b) \tau^{-1}\right) \tau-\sim b+(\sim-b),
\end{aligned}
$$

so (2) holds.

(3): This is obtained from (2) by replacing $a$ with $a \tau$ and $b$ with $b \tau$.

Lemma 3.2. Let $a, b \in U^{*}$ with $a \neq b$ and $\mu_{a}=\mu_{-b}$. Then

(1) $\left(a \tau^{-1}-b \tau^{-1}\right) \tau-\left((-b) \tau^{-1}-(-a) \tau^{-1}\right) \tau=\sim b-\sim-a$;

(2) $(a-b) \tau-((\sim b)-(\sim a)) \tau=\sim(b \tau)-\sim-(a \tau)$; in particular, 
(3) $(a-b) \mu_{b}-((\sim b)-(\sim a)) \mu_{b}=a-\sim b$;

(4) if $\sim a=-a$ and $\sim b=-b$, then $a=-b$.

Proof. (1): By equation (1.4),

$$
\left(a \tau^{-1}-b \tau^{-1}\right) \tau=(a-b) \mu_{b}+(\sim b)
$$

and

$$
\left((-b) \tau^{-1}-(-a) \tau^{-1}\right) \tau=(a-b) \mu_{-a}+(\sim-a)=(a-b) \mu_{b}+(\sim-a) ;
$$

subtracting we get (1).

(2): This part is obtained from (1) by replacing $a$ with $a \tau$ and $b$ with $b \tau$.

(3): By (2), with $\tau=\mu_{b}$ we have

$$
(a-b) \mu_{b}-((\sim b)-(\sim a)) \mu_{b}=\sim\left(b \mu_{b}\right)-\sim-\left(a \mu_{b}\right)=\sim\left(b \mu_{b}\right)-\sim-\left(a \mu_{-a}\right) .
$$

But by Lemma 2.3, $b \mu_{b}=\sim-\sim b$ and $a \mu_{-a}=-\sim-a$, so (3) holds.

(4): If $\sim a=-a$ and $\sim b=-b$, then by (3), $a-\sim b=0$, so $a=-b$.

\section{Consequences of the Equality $\mu_{a}=\mu_{b}$}

Throughout this section $a, b \in U^{*}$ are such that $a \neq b$ and $\mu_{a}=\mu_{b}$. We denote

$$
\mu:=\mu_{a}=\mu_{b} .
$$

Lemma 4.1. We have

(1) $\mu_{a \mu^{-1}-b \mu^{-1}}=\mu_{a-b}$;

(2) $\mu_{a-b}=\mu_{\sim a-\sim b}$;

(3) $\mu_{a \mu-b \mu}=\mu_{b-a}$;

(4) $\mu_{a-b}^{\mu^{2}}=\mu_{b-a}$.

Proof. (1): This is Lemma 2.5(1).

(2): This follows from Lemma 2.5(2), by interchanging $a$ and $b$ and using the fact that $U$ is commutative.

(3): By (2) with $-a$ in place of $a$ and $-b$ in place of $b$ we get

$$
\mu_{b-a}=\mu_{-a-(-b)}=\mu_{\sim-a-\sim-b} .
$$

Replacing $b$ with $\sim b$ and $a$ with $\sim a$ in (*) and using (2) we get

$$
\mu_{b-a}=\mu_{\sim b-\sim a} \stackrel{(*)}{=} \mu_{\sim-\sim a-\sim-\sim b} .
$$

By Lemma 2.3, $\sim-\sim a=a \mu$ and $\sim-\sim b=b \mu$, so (3) holds.

(4): Let $x:=a \mu$ and $y:=b \mu$. Then $x \neq y$ and $\mu_{x}=\mu^{-1}=\mu_{y}$. We have

$$
\mu_{a \mu^{2}-b \mu^{2}}=\mu_{x \mu-y \mu}=\mu_{x \mu_{x}^{-1}-y \mu_{x}^{-1}} \stackrel{(1)}{=} \mu_{x-y},
$$

so (4) follows from (3).

Lemma 4.2. We have

(1) $\mu^{\mu_{b-a}}=\mu_{((\sim a)-(\sim b))-(a-b)}$;

(2) $\mu^{\mu_{a-b}}=\mu_{(a-b)-((\sim a)-(\sim b))}$;

(3) $\mu^{\mu_{a-b}^{2}}=\mu^{-1}$;

(4) if $a \mu^{2}=a$ and $b \mu^{2}=b$, then $\mu^{2}=1$ 
Proof. (1): Using Lemma 3.1(3), equation (1.4) and Lemma 4.1(2), we get

$$
\begin{aligned}
\mu & =\mu_{((a-b) \tau-((\sim a)-(\sim b)) \tau) \tau^{-1}} \\
& =\mu_{(\sim b)-(\sim a)} \mu_{((\sim a)-(\sim b))-(a-b)} \mu_{a-b} \\
& =\mu_{b-a} \mu_{((\sim a)-(\sim b))-(a-b)} \mu_{a-b}
\end{aligned}
$$

This shows (1).

(2): Set $x:=\sim a-\sim b$ and $y:=a-b$. By Lemma 4.1(2), $\mu_{x}=\mu_{y}$, and by (1), $\mu=\mu_{x-y}^{\mu_{x}}$. Further, by Lemma 4.1(4), $\mu_{x-y}^{\mu_{x}^{2}}=\mu_{y-x}$. Hence, $\mu^{\mu_{x}}=\mu_{x-y}^{\mu_{x}^{2}}=\mu_{y-x}$ as asserted.

(3): By (1) and (2),

$$
\mu^{\mu_{b-a}}=\mu_{((\sim a)-(\sim b))-(a-b)}=\left(\mu_{(a-b)-((\sim a)-(\sim b))}\right)^{-1}=\left(\mu^{-1}\right)^{\mu_{a-b}},
$$

and (3) follows.

(4): Assume that $a \mu^{2}=a$ and $b \mu^{2}=b$. By Lemma 4.1(4) we get that

$$
\mu_{a-b}=\mu_{a \mu^{2}-b \mu^{2}}=\mu_{a-b}^{\mu^{2}}=\mu_{b-a},
$$

so $\mu_{a-b}^{2}=1$. Then, by $(3), \mu^{2}=1$.

Lemma 4.3. Let $\nu:=\mu_{a-b}$. Then

(1) $\mu^{\nu^{2}}=\mu^{-1}$ and $\nu^{\mu^{2}}=\nu^{-1}$;

(2) $\mu^{8}=\nu^{8}=1$;

(3) $\mu$ is an involution iff $\nu$ is an involution;

(4) $a \mu^{4}=a$ and $b \mu^{4}=b$.

Proof. (1): By Lemma 4.1(4), $\nu^{\mu^{2}}=\nu^{-1}$, and by Lemma 4.2(3), $\mu^{\nu^{2}}=\nu^{-1}$.

(2): By (1), $\nu^{4}=\mu^{-2} \nu^{-2} \mu^{2} \nu^{2}=\mu^{-4}$ and $\nu^{4}=\mu^{2} \nu^{-2} \mu^{-2} \nu^{2}=\mu^{4}$. It follows that $\mu^{8}=1$ and then also $\nu^{8}=1$.

(3): This is immediate from (1).

(4): Assume that $a \mu^{4} \neq a$. Then we can take $a \mu^{4}$ in place of $b$ in (1) to get that $\mu^{2}$ inverts $\rho:=\mu_{a \mu^{4}-a}$ and hence $\mu^{4}$ centralizes $\rho$. It follows that

$$
\rho=\rho^{\mu^{4}}=\mu_{a \mu^{8}-a \mu^{4}}=\mu_{a-a \mu^{4}}=\rho^{-1},
$$

where we have used the fact that $\mu^{8}=1$. Thus $\rho^{2}=1$, so by $(3), \mu^{2}=1$, contradicting $a \mu^{4} \neq a$. Hence $a \mu^{4}=a$ and by symmetry $b \mu^{4}=b$.

Proposition 4.4. We have

(1) $\sim-a+a \mu^{2}-\sim a=-\left(\sim-\left(b \mu^{2}\right)+b-\sim\left(b \mu^{2}\right)\right)$;

(2) if $\sim a \neq-a$, then $\left(\sim-a+a \mu^{2}-\sim a\right) \cdot 2=0$.

Proof. (1): By Lemma 4.3(4),

$$
(a-b) \mu^{2}=a \mu^{2}-b \mu^{2}=a \mu^{-2}-b \mu^{-2}=(a-b) \mu^{-2} .
$$

It follows that

$$
\begin{aligned}
{\left[(a-b) \mu-(b-a) \mu^{-1}\right] \mu^{2} } & =(a-b) \mu^{-1}-(b-a) \mu \\
& =-\left[(b-a) \mu-(a-b) \mu^{-1}\right],
\end{aligned}
$$

So

$$
\left[(a-b) \mu-(b-a) \mu^{-1}\right] \mu^{2}=-\left[(b-a) \mu-(a-b) \mu^{-1}\right] .
$$


By equation (4.1) and Lemma 3.1(1) we get

$$
(\sim-b+a-\sim b) \mu^{2}=-\sim-a-b+\sim a .
$$

Since $(-x) \mu^{2}=-\left(x \mu^{2}\right)$ and $(\sim x) \mu^{2}=\sim\left(x \mu^{2}\right)$, for all $x \in U^{*}$, and since $\mu^{2} \in$ Aut $(U)$, equation (4.2) implies (1).

(2): In equation (4.2), take the minus of both sides and then take $b=-\sim a$. This gives

$$
\begin{aligned}
\sim-a-(\sim a) \cdot 2 & =(\sim-\sim a-a \cdot 2) \mu^{2} & & \\
& =\sim-\sim a \mu^{2}-\left(a \mu^{2}\right) \cdot 2 & & \left(\text { because } \mu^{2} \in \operatorname{Aut}(U)\right) \\
& =\sim-\sim a \mu^{-2}-\left(a \mu^{2}\right) \cdot 2 & & \text { (by Lemma 4.3(4)) } \\
& =-\sim-a-\left(a \mu^{2}\right) \cdot 2 & & \text { (by Lemma 2.3 (10)). }
\end{aligned}
$$

We thus see that $-\left(a \mu^{2}\right) \cdot 2=(\sim-a-\sim a) \cdot 2$, so (2) holds.

Lemma 4.5. Assume that $a \mu=-a$ and $b \mu=-b$. Then

(1) $a=-\sim-b$;

(2) if $\sim a \neq-a$ and $(-\sim a) \mu=\sim a$, then $a \cdot 2=0$.

Proof. We have

$$
(b-a) \mu^{-1}=(a \mu-b \mu) \mu^{-1}=(a-b) \mu+\sim b .
$$

Using Lemma 3.1(1) we get that

$$
-\sim b=(a-b) \mu-(b-a) \mu^{-1}=\sim-b+a-\sim b .
$$

This shows (1). Then under the hypotheses of (2) we may take $b=-\sim a$ in (1) to get $a=-a$, so (2) holds.

Lemma 4.6. Let $a \in U^{*}$. Then $V_{a} \nsubseteq\{0, a,-a\}$ iff there exists $c \in V_{a}^{*}$ such that $\sim c \neq-c$.

Proof. Suppose $V_{a} \subseteq\{0, a,-a\}$. By Lemma 2.4, $\sim a=-a$, so also $\sim(-a)=-(-a)$ and hence there exists no $c \in V_{a}^{*}$ such that $\sim c \neq-c$.

Conversely, assume that $V_{a} \nsubseteq\{0, a,-a\}$. We show that there exists $c \in V_{a}^{*}$ such that $\sim c \neq-c$. If $\sim a \neq-a$, take $c=a$. So assume that $\sim a=-a$ and let $c \in V_{a}^{*}$ such that $c \notin\{a,-a\}$. Suppose that $\sim c=-c$ and let $x=-c$. Then $x \neq a$ and $\mu_{a}=\mu_{-x}$. By Lemma 3.2 (4), $x=-a$, a contradiction.

\section{Consequences of the equality $\mu_{a}=\mu_{-b}$}

In this section, $a, b \in U^{*}$ are such that $a \neq b$ and $\mu_{a}=\mu_{-b}$.

Lemma 5.1. We have

(1) $\mu_{a-b}=\mu_{a \mu_{a}^{-1}-b \mu_{a}^{-1}} \mu_{a}^{2}=\mu_{a}^{2} \mu_{a \mu_{a}-b \mu_{a}}$;

(2) $\mu_{a-b}=\mu_{\sim b-\sim a}$;

(3) $\mu_{a-b}$ commutes with $\mu_{a}^{2}$.

Proof. (1): This is Lemma 2.6. 
(2): Replacing $a$ with $\sim a$ and $b$ with $\sim b$ in (1) we must replace $\mu_{a}^{-1}$ with $\mu_{\sim a}^{-1}=\mu_{a}$, so we get

$$
\begin{aligned}
\mu_{\sim a-\sim b} & =\mu_{(\sim a) \mu_{a}-(\sim b) \mu_{a}} \mu_{-a}^{2} \\
& =\mu_{b \mu_{a}-a \mu_{a}} \mu_{-a}^{2} \\
& \stackrel{(1)}{=} \mu_{a-b}^{-1}=\mu_{b-a} .
\end{aligned}
$$

Taking inverses we get $(2)$.

(3): Suppose that $a \mu_{a}^{2} \neq a$. Then $b:=a \mu_{a}^{2}$ satisfies $\mu_{a}=\mu_{b}$, so we can apply Lemma 4.3. (4) to get $a \mu_{a}^{4}=a$. Thus we always have

$$
a \mu_{a}^{4}=a, \quad \text { for all } a \in U^{*} .
$$

We claim that

$$
\mu_{a-b}=\mu_{a \mu_{-a}^{2}-b \mu_{a}^{2}}
$$

Indeed by (2) we have $\mu_{a-b}=\mu_{-b-(-a)}=\mu_{(\sim-a)-(\sim-b)}$, so

$$
\mu_{a-b}=\mu_{(\sim-a)-(\sim-b) .}
$$

Applying equation (5.3) three times we get that $\mu_{a-b}=\mu_{(\sim-\sim-\sim-a)-(\sim-\sim-\sim-b)}$. By Lemma 2.3(10) we get

$$
\mu_{a-b}=\mu_{a \mu_{-a}^{2}-b \mu_{-b}^{2}}=\mu_{a \mu_{-a}^{2}-b \mu_{a}^{2}}
$$

so equation (5.2) holds. But now we have

$$
\begin{aligned}
\mu_{a-b} & =\mu_{a \mu_{-a}^{2}-b \mu_{a}^{2}} \quad(\text { this is equation (5.2) }) \\
& \left.=\mu_{a \mu_{a}^{2}-b \mu_{a}^{2}} \quad \text { (by equation (5.1) }\right) \\
& =\mu_{a-b}^{\mu_{a}^{2}} .
\end{aligned}
$$

\section{One more Lemma For the EQUALity $\mu_{a}=\mu_{b}$}

Throughout this section $a, b \in U^{*}$ are such that $\mu_{a}=\mu_{b}$. In this section we add one more lemma to section 4. As in section 4 we let

$$
\mu:=\mu_{a}=\mu_{b}
$$

Lemma 6.1. Assume that $a \neq-b$. Then

(1) $\mu_{a+b}=\mu_{\sim-a-\sim b}$;

(2) $\mu_{a+b}=\mu_{(-a) \mu^{-1}-b \mu}$;

(3) $\mu_{a+b}=\mu_{a \mu^{-1}-(-b) \mu^{-1}} \mu^{2}=\mu^{2} \mu_{a \mu-(-b) \mu}$;

(4) $\mu_{a+b \mu^{2}}=\mu_{a+b}^{-1} \mu^{2}=\mu^{2} \mu_{a+b}^{-1}$.

Proof. (1): We have $\mu_{a}=\mu_{-(-b)}$, so we can apply Lemma 5.1(2) with $-b$ in place of $b$ to get

$$
\mu_{a+b}=\mu_{a-(-b)}=\mu_{\sim-b-\sim a} .
$$

Interchanging $a$ and $b$ we get (1).

(2): Replacing in (1) $a$ with $\sim-a$ and $b$ with $-\sim b$ (notice that $\sim-a \neq-(-\sim b)$ ) we get that $\mu_{\sim-a-\sim b}=\mu_{\sim-\sim-a-\sim-\sim b}$. But by Lemma 2.3 (6) and $(7), \sim-\sim-a=$ $(-a) \mu^{-1}$ and $-\sim-\sim b=-(b \mu)$. This shows $(2)$. 
(3): As in the proof of (1) we can apply Lemma 5.1(1) with $-b$ in place of $b$ to get

$$
\mu_{a+b}=\mu_{a-(-b)}=\mu_{a \mu^{-1}-(-b) \mu^{-1}} \mu^{2}=\mu^{2} \mu_{a \mu-(-b) \mu} .
$$

(4): Note that if $a=-b \mu^{2}$, then $\mu_{a}=\mu_{-b \mu^{2}}=\mu_{-b}=\mu_{-a}$, and then $\mu^{2}=1$, so $a=-b$, which is contrary to our assumption. Hence in (3) we can take $b \mu^{2}$ in place of $b$. Notice that $-b \mu^{2}=(-b) \mu^{2}$; consequently we get

$$
\mu_{a+b \mu^{2}}=\mu_{a \mu^{-1}-(-b) \mu^{2} \mu^{-1}} \mu^{2}=\mu_{a \mu^{-1}-(-b) \mu} \mu^{2} .
$$

Notice now that $a \mu^{-1}=-\sim-a$ and $-(-b) \mu=\sim b$. Hence $a \mu^{-1}-(-b) \mu=$ $-(\sim-a-\sim b)$. Using (1) we get

$$
\mu_{a+b \mu^{2}}=\mu_{-(\sim-a-\sim b)} \mu^{2}=\mu_{a+b}^{-1} \mu^{2} .
$$

This shows the first equality in (4), and the second equality follows from Lemma $5.1(3)$.

\section{Consequences of the hypothesis $\mu_{a}^{2}=1$}

Throughout this section $a \in U^{*}$ is such that

$$
\mu_{a}^{2}=1
$$

We recall from equation (1.1) the notation $V_{a}$.

Theorem 7.1. Assume that $\sim a \neq-a$, and set $t:=\sim-a+a-\sim a$. Then

(1) $t \cdot 2=0$;

(2) $\sim-v+v-\sim v=t$, for all $v \in V_{a}$;

(3) $V_{a}$ is a root subgroup of $U$.

Proof. By Proposition 4.4(2), $t \cdot 2=0$, and then (2) follows from Proposition 4.4(1) and from the hypothesis that $\mu_{a}^{2}=1$.

Let $u, v \in V_{a}$ with $u \neq v$. By hypothesis, $-u \in V_{a}$. By (2),

$$
\sim-u+u-\sim u=t=\sim-v+v-\sim v,
$$

so

$$
(u-v)-(\sim u-\sim v)=\sim-v-\sim-u .
$$

Using Lemma 4.1(2) with $\sim-v$ in place of $a$ and $\sim-u$ in place of $b$ we see that

$$
\mu_{(u-v)-(\sim u-\sim v)}=\mu_{\sim-v-\sim-u}=\mu_{u-v} .
$$

But now by Lemma $4.2(2)$ with $u, v$ in place of $a, b$ we get that

$$
\mu_{u}^{\mu_{u-v}}=\mu_{(u-v)-(\sim u-\sim v)}=\mu_{u-v},
$$

so we see that $\mu_{a}=\mu_{u}=\mu_{u-v}$ and hence $u-v \in V_{a}$. This shows that $V_{a}$ is a subgroup of $U$. Also

$$
\mu_{u \mu_{v}}=\mu_{-v} \mu_{-u} \mu_{v}=\mu_{-a}=\mu_{a},
$$

so $u \mu_{v} \in V_{a}^{*}$. By definition (see [S1], section 3) $V_{a}$ is a root subgroup of $U$.

Corollary 7.2. Assume that $V_{a} \nsubseteq\{0, a,-a\}$. Then $V_{a}$ is a root subgroup of $U$.

Proof. By Lemma 4.6 there exists $b \in V_{a}^{*}$ such that $\sim b \neq-b$, so the corollary follows from Theorem 7.1 . 
Here is another very useful result:

Lemma 7.3. Let $a, b \in U^{*}$ with $a \neq b$ and let $c:=\left(a \tau^{-1}-b \tau^{-1}\right) \tau$. If $\mu_{a}, \mu_{b}, \mu_{c}$ are involutions, then $\mu_{a-b}=\mu_{\sim a-\sim b}$.

Proof. Since $(\sim a) \tau^{-1}=-\left(a \tau^{-1}\right)$ and $(\sim b) \tau^{-1}=-\left(b \tau^{-1}\right)$, we have

$$
\begin{gathered}
\left(a \tau^{-1}-b \tau^{-1}\right) \tau=\left((\sim b) \tau^{-1}-(\sim a) \tau^{-1}\right) \tau \\
\left.=\left(-\left((\sim a) \tau^{-1}-(\sim b) \tau^{-1}\right)\right) \tau=\sim\left((\sim a) \tau^{-1}-(\sim b) \tau^{-1}\right) \tau\right] .
\end{gathered}
$$

Since $\mu_{c}$ is an involution we see that

$$
\mu_{c}=\mu_{\left((\sim a) \tau^{-1}-(\sim b) \tau^{-1}\right) \tau} .
$$

Using equation (1.4) we get

$$
\mu_{-b} \mu_{b-a} \mu_{a}=\mu_{\left(a \tau^{-1}-b \tau^{-1}\right) \tau}=\mu_{\left((\sim a) \tau^{-1}-(\sim b) \tau^{-1}\right) \tau}=\mu_{-\sim b} \mu_{\sim b-\sim a} \mu_{\sim a} .
$$

It remains to observe that $\mu_{-b}=\mu_{-\sim b}$ and $\mu_{a}=\mu_{\sim a}$, because $\mu_{a}$ and $\mu_{b}$ are involutions.

\section{The CASE WHERE AlL $\mu$-MAPS ARE INVOLUTiOnS}

In this section we assume that all $\mu$-maps are involutions. Our aim in this section is to prove the following theorem:

Theorem 8.1. Let $\mathbb{M}(U, \tau)$ be a Moufang set and assume that

(i) $U$ is abelian.

(ii) $\mu_{a}$ is an involution, for all $a \in U^{*}$.

Then either $\mathbb{M}(U, \tau)$ is sharply 2-transitive, or $\mathbb{M}(U, \tau)$ is special.

We start with

Lemma 8.2. Let $b \in U^{*}$ and assume that $\mu_{b}^{2}=1$ and that $\sim b=-b$. Suppose $b \cdot 2 \neq 0$ and that $V_{b \cdot 2} \nsubseteq\{0, b \cdot 2,-b \cdot 2\}$. Then $\mu_{b}=\mu_{b \cdot 2}$.

Proof. By Lemma 3.1(3), with $b$ in place of $a,-b$ in place of $b$, and $\mu_{b \cdot 2}$ in place of $\tau$ we see that

$$
\left((b \cdot 2) \mu_{b \cdot 2}-(-b \cdot 2) \mu_{b \cdot 2}\right) \mu_{-b \cdot 2}=b .
$$

But by Corollary 7.2, $V_{b \cdot 2}$ is a root subgroup of $U$ and hence the LHS of the above equality is in $V_{b \cdot 2}$. It follows that $b \in V_{b \cdot 2}$ as asserted.

Proposition 8.3. Assume that there exists $a \in U^{*}$ such that $\sim a=-a$. Then $\mathbb{M}(U, \tau)$ is either special or sharply 2-transitive.

Proof. We claim that

$$
\text { if } b \in U^{*} \text { and } \sim b \neq-b \text {, then } \mu_{b}=\mu_{a} .
$$

Suppose that (8.1) holds. Suppose also that $\mathbb{M}(U, \tau)$ is not special. We show that if $b \in U^{*}$ is such that $\sim b=-b$, then $\mu_{b}=\mu_{a}$. Indeed, let $c \in U^{*}$ with $\sim c \neq-c$. Then, by (8.1), $\mu_{a}=\mu_{c}=\mu_{b}$.

Next we claim that $\mu_{b}=\mu_{a}$, for all $b \in U^{*}$. So let $b \in U^{*}$. If $\sim b=-b$, then, by the previous paragraph of the proof, $\mu_{b}=\mu_{a}$, while if $\sim b \neq-b$, then $\mu_{b}=\mu_{a}$, by (8.1). We may thus conclude that $\mathbb{M}(U, \tau)$ is sharply 2-transitive as asserted.

Hence it remains to prove (8.1). So let $b \in U^{*}$ such that $\sim b \neq-b$. We want to show that $\mu_{a}=\mu_{b}$. Suppose that $\sim b=b$. Notice that $\sim(-b) \neq-(-b)$ and if $\sim(-b)=-b$, then by Lemma 2.1 $(2), \sim b=b=-b$, a contradiction. Hence, after 
replacing $b$ with $-b$ if necessary, we may assume without loss that $\sim b \neq b$. By Theorem 7.1 ,

$$
V_{b} \text { is a subgroup of } U \text {. }
$$

By Lemma 7.3, $\mu_{a-b}=\mu_{-a-\sim b}$. Assume first that $a-b=-a-\sim b$. Then $a \cdot 2=$ $b-\sim b \in V_{b}^{*}$ (because $\sim b \neq b$ ), so $V_{a \cdot 2}=V_{b}$. Further, since $b \in V_{a \cdot 2}$ and $\sim b \neq-b$, Lemma 4.6 implies that $V_{a \cdot 2} \nsubseteq\{0, a \cdot 2,-a \cdot 2\}$. We can thus apply Lemma 8.2 to get $\mu_{a}=\mu_{a \cdot 2}=\mu_{b-\sim b}=\mu_{b}$. Assume next that $a-b \neq-a-\sim b$. We show that $V_{a-b}$ is a subgroup of $U$. For that it suffices to show that $V_{a-b} \nsubseteq\{0, a-b, b-a\}$ (see Corollary 7.2). Since $-a-\sim b \in V_{a-b}^{*}$, it suffices to show that $a-b \neq-a-\sim b \neq b-a$. But we are assuming that $a-b \neq-a-\sim b$ and since $\sim b \neq-b$, we have $-a-\sim b \neq b-a$, so $V_{a-b}$ is a subgroup of $U$. It follows that

$$
\mu_{a-b}=\mu_{a-b+(-a-\sim b)}=\mu_{-b-\sim b}=\mu_{b},
$$

where the last equality is since $V_{b}$ is a subgroup of $U$. But since $V_{b}$ is a subgroup of $U$ the equality $\mu_{a-b}=\mu_{b}$ implies that $\mu_{b}=\mu_{a-b+b}=\mu_{a}$, as asserted.

We are now in a position to prove Theorem 8.1 .

Proof of Theorem 8.1. Assume toward a contradiction that $\mathbb{M}(U, \tau)$ is neither special nor sharply 2-transitive. In view of Proposition 8.3 and Theorem 7.1 we have

$$
\sim a \neq-a, \text { and } V_{a} \text { is a subgroup of } U, \quad \forall a \in U^{*} .
$$

Let $a, b \in U^{*}$ with $a \neq b$. We show that equation (8.2) leads to the conclusion that $\mu_{a}=\mu_{b}$, thereby obtaining our desired contradiction; indeed this implies that $\mu_{x}=\mu_{y}$, for all $x, y \in U^{*}$, and hence $\mathbb{M}(U, \tau)$ is a sharply 2-transitive Moufang set.

Let

$$
c:=\left(a \tau^{-1}-b \tau^{-1}\right) \tau \quad \text { and } \quad d:=\left((-a) \tau^{-1}-(-b) \tau^{-1}\right) \tau .
$$

Notice that $d=\left(\sim\left(a \tau^{-1}\right)-\sim\left(b \tau^{-1}\right)\right) \tau$. By Lemma 7.3, $\mu_{a \tau^{-1}-b \tau^{-1}}=\mu_{\sim\left(a \tau^{-1}\right)-\sim\left(b \tau^{-1}\right)}$ and it follows that

$$
\mu_{d}=\mu_{c}
$$

Next we claim that

$$
c=(a-b) \mu_{b}+\sim b=(\sim b-\sim a) \mu_{a}+a .
$$

The first equality in (8.4) is by equation (1.4). Also since $(\sim a) \tau^{-1}=-\left(a \tau^{-1}\right)$ and $(\sim b) \tau^{-1}=-\left(b \tau^{-1}\right)$ we get, using again equation (1.4), that

$$
c=\left(a \tau^{-1}-b \tau^{-1}\right) \tau=\left((\sim b) \tau^{-1}-(\sim a) \tau^{-1}\right) \tau=(\sim b-\sim a) \mu_{a}+a,
$$

so equation (8.4) holds. Note now that $\sim c=\left(b \tau^{-1}-a \tau^{-1}\right) \tau$, so interchanging $a$ and $b$ in (8.4) gives

$$
\sim c=(b-a) \mu_{a}+\sim a=(\sim a-\sim b) \mu_{b}+b .
$$

Also replacing $a$ by $-a$ and $b$ by $-b$ in equation (8.4) gives

$$
d=(b-a) \mu_{b}+\sim-b=(\sim-b-\sim-a) \mu_{a}-a .
$$

We now divide the proof into two cases:

Case 1. $c+d=0$. 
Since $d=-c$, equations (8.4) and (8.6) imply that

$$
-(a-b) \mu_{b}-\sim b=(b-a) \mu_{b}+\sim-b
$$

or

$$
-(a-b) \mu_{b}-(b-a) \mu_{b}=\sim-b+\sim b .
$$

Notice that $\sim-b+\sim b \neq 0$; otherwise $\sim-\sim-b=b$, and then $\sim-\sim-\sim-b=\sim-b$. But by Lemma 2.3, $\sim-\sim-\sim-b=b$, so we get that $\sim-b=b$ and then $\sim b=-b$, contradicting (8.2).

Returning to equation (8.7), consider $x:=-(a-b) \mu_{b}-(b-a) \mu_{b}$. Working in the group $V_{(a-b) \mu_{b}}$ (see equation (8.2)) we see that $\mu_{x}=\mu_{(a-b) \mu_{b}}$. However working in the group $V_{b}$ shows that $\mu_{\sim-b+\sim b}=\mu_{b}$, and we conclude from equation (8.7) that

$$
\mu_{(a-b) \mu_{b}}=\mu_{b} .
$$

But by equation (1.2), $\mu_{(a-b) \mu_{b}}=\mu_{b} \mu_{b-a} \mu_{b}$, so equation (8.8) implies that

$$
\mu_{b-a}=\mu_{b} .
$$

Working in the group $V_{b}$ using equation (8.9) we see now that $\mu_{a}=\mu_{b}$.

Case 2. $c+d \neq 0$.

By equations (8.4) and (8.6),

$$
c+d=(\sim b-\sim a) \mu_{a}+(\sim-b-\sim-a) \mu_{a} .
$$

Working in the group $V_{c}$ using equation (8.3) we see that $\mu_{c+d}=\mu_{c}$. Working in the group $V_{(b-a) \mu_{a}}$ noticing that by Lemma 7.3 .

$$
\mu_{(\sim b-\sim a) \mu_{a}}=\mu_{(b-a) \mu_{a}}=\mu_{(\sim-b-\sim-a) \mu_{a}},
$$

we see that for $x:=(\sim b-\sim a) \mu_{a}+(\sim-b-\sim-a) \mu_{a}$ we have $\mu_{x}=\mu_{(b-a) \mu_{a}}$. We can now conclude that

$$
\mu_{c}=\mu_{(b-a) \mu_{a}} .
$$

Arguing as above using equation (8.5) (which implies that $\mu_{c}=\mu_{\sim c}=\mu_{(b-a) \mu_{a}+\sim a}$ ) and working in the group $V_{c}$ we obtain that $\mu_{(b-a) \mu_{a}}=\mu_{a}$. It follows that $\mu_{a-b}=\mu_{a}$ and arguing in the group $V_{a}$ we get that $\mu_{a}=\mu_{b}$. This completes the proof of the theorem.

\section{THE PROOF THAT IF $\sim a \neq-a$, THEN $\mu_{a}^{2}=1$}

Our aim in this section is to show

Theorem 9.1. If $a \in U^{*}$ is such that $\sim a \neq-a$, then $\mu_{a}^{2}=1$.

This will be done in a series of lemmas. Thus, throughout this section,

$$
a \in U^{*} \text { is an element such that } \sim a \neq-a \text {. }
$$

We start with

Lemma 9.2. We may (and we will) assume that $\mu_{a}^{4}=1$.

Proof. Consider the root subgroup $V \subseteq U$ of all elements fixed by $\mu_{a}^{4}$. By Lemma 4.3(4), $a \in V^{*}$ and hence also $\sim a=-(-a) \mu_{a} \in V^{*}$. Hence the hypothesis $\sim a \neq-a$ holds in $\mathbb{M}(V, \tau)$ as well. Suppose the theorem holds for $\mathbb{M}(V, \tau)$; then $\mu_{a}^{2}$ is the identity on $V$, so, in particular, $a \mu_{a}^{2}=a$ and $(-\sim a) \mu_{a}^{2}=-\sim a$. But then by Lemma 4.2(4) (with $b=-\sim a), \mu_{a}^{2}=1$ and we are done. 
We now assume that Theorem 9.1 is false; that is, we assume that $\mu_{a}^{2} \neq 1$, and our aim is to obtain a contradiction. Notice that $a \cdot 2 \neq 0$, else $\mu_{a}=\mu_{-a}$ and then $\mu_{a}^{2}=1$.

Lemma 9.3. We have

(1) $a \mu_{a}^{2} \neq a$;

(2) $\mu_{a \cdot 2}=\mu_{\sim-a-\sim a}$;

(3) $\mu_{a+a \mu_{a}^{2}}=\mu_{a \cdot 2}^{-1} \mu_{a}^{2}$;

(4) $a \mu_{a}^{2} \neq-a$.

Proof. (1): Suppose $a \mu_{a}^{2}=a$. Then also $(-\sim a) \mu_{a}^{2}=-\sim a$. But then by Lemma 4.2 (4) with $b=-\sim a$ we get that $\mu_{a}^{2}=1$, a contradiction.

(2) and (3): Just take $a=b$ in Lemma 6.1, (1) and (4), respectively.

(4): If $a \mu_{a}^{2}=-a$, then $\mu_{a}=\mu_{a \mu_{a}^{2}}=\mu_{-a}$, so $\mu_{a}^{2}=1$, a contradiction.

Lemma 9.4. Set $x=a+a \mu_{a}^{2}$. Then

(1) $-a \mu_{a}^{2}=\sim-a-\sim a$;

(2) $\mu_{a \cdot 2}=\mu_{a}^{-1}$;

(3) $x \neq 0$;

(4) $\mu_{x}=\mu_{-a}$;

(5) $\sim x=-x$;

(6) for $y=\sim a+(\sim a) \mu_{a}^{2}$ we have $x=-y$.

Proof. (1): In Lemma 3.1(1) we take $a \mu_{a}^{2}$ in place of $a$ and $a$ in place of $b$. We get

$$
\left(a \mu_{a}^{2}-a\right) \mu_{a}-\left(a-a \mu_{a}^{2}\right) \mu_{-a}=\sim-a+a \mu_{a}^{2}-\sim a .
$$

Notice now that $\left(a \mu_{a}^{2}-a\right) \mu_{a}=\left(a-a \mu_{a}^{2}\right) \mu_{-a}$, because $\mu_{a}^{4}=1$, and since $\mu_{a}^{2} \in$ Aut $(U)$. Hence the LHS of equation (9.1) is zero, so the RHS is also zero.

(2), (3) and (4): Part (2) follows from (1) and Lemma 9.3) (2), and (3) comes from Lemma 9.3(4). By Lemma 9.3(3) and by (2),

$$
\mu_{a+a \mu_{a}^{2}}=\mu_{-a \cdot 2} \mu_{a}^{2}=\mu_{a}^{3}=\mu_{-a},
$$

so (4) holds.

(5): Assume that $\sim x \neq-x$. We claim that $\mu_{x}^{2}=1$. For this notice that by (4), $x \mu_{x}^{2}=x \mu_{a}^{2}=x$. Also, $(\sim-x) \mu_{x}^{2}=\sim-x$. Thus applying Lemma 4.2(4) (with $a=x$ and $b=\sim-x)$, we see that $\mu_{x}^{2}=1$. But then by $(4), \mu_{a}^{2}=\mu_{-a}^{2}=\mu_{x}^{2}=1$, a contradiction.

(6): Replacing $a$ with $\sim a$ and arguing as in (5) we see that $\sim y=-y$ and by (4), $\mu_{y}=\mu_{-\sim a}=\mu_{a}$. Notice that if $x=y$, then $\mu_{-a}=\mu_{x}=\mu_{y}=\mu_{a}$, so $\mu_{a}^{2}=1$, a contradiction. Now taking $x$ in place of $a$ and $y$ in place of $b$ in Lemma [3.2(4), we see that (6) holds.

We are now ready to obtain a contradiction and thereby to prove Theorem 9.1 .

Proof of Theorem 9.1. First notice that by Lemma 9.4),

$$
a \mu_{a}^{2}=-\sim-a+\sim a .
$$

By Lemma 9.4(6) we have $a+a \mu_{a}^{2}=-\left(\sim a+(\sim a) \mu_{a}^{2}\right)$ and hence

$$
(a+\sim a) \mu_{a}^{2}=-(a+\sim a) .
$$

Next, applying Lemma 3.1(1) with $b=-\sim a$ gives

$$
(a+\sim a) \mu_{a}-(-(a+\sim a)) \mu_{-a}=a \cdot 2-\sim-\sim a .
$$


But by equation (9.3),$(a+\sim a) \mu_{a}=(-(a+\sim a)) \mu_{-a}$, so from equation (9.4) we see that

$$
a \cdot 2=\sim-\sim a .
$$

Replacing $a$ with $-\sim-a$ in equation (9.5) (note that this is possible because $\sim b \neq$ $-b$, for $b=-\sim-a)$, and using Lemma 2.3(10) we get that

$$
a \mu_{a}^{2}=(-\sim-a) \cdot 2 .
$$

But now equation (9.2) shows that $-\sim-a=\sim a$, or $\sim-\sim-a=a$. By Lemma 2.3(6) this shows that $(-a) \mu_{-a}=a$, so $a \mu_{a}=-a$. Replacing $a$ with $-\sim a$ we get that $(-\sim a) \mu_{a}=\sim a$. Since we are assuming that $\sim a \neq-a$, we get from Lemma 4.5) (2) that $a \cdot 2=0$, a contradiction.

Corollary 9.5. Let $b \in U^{*}$ such that $V_{b} \nsubseteq\{0, b,-b\}$ and set $t_{b}:=\sim-b+b-\sim b$. Then

(1) there exists $c \in V_{b}$ such that $\sim c \neq-c$;

(2) $\mu_{b}^{2}=1$;

(3) $V_{b}$ is a root subgroup of $U$;

(4) $t_{b} \cdot 2=0$ and $t_{b}=\sim-v+v-\sim v$, for all $v \in V_{b}^{*}$.

Proof. Part (1) holds by Lemma 4.6, By (1) and Theorem 9.1 (2) holds. By (2) and Corollary 7.2. (3) holds. Finally, by Theorem 7.1(1 and 2), $t_{b}=\sim-c+c-\sim c$ satisfies $t_{b} \cdot 2=0$, and $t_{b}=\sim-v+v-\sim v$, for all $v \in V_{b}^{*}$.

\section{SOME AUXILIARY LEMMAS}

In this section we prove a few lemmas that will help us with the proof of the Main Theorem. The purpose of the first three lemmas is to deal with elements of order 3 .

Lemma 10.1. Let $a \in U^{*}$ such that $\mu_{a}^{2}=1$. Assume that $a \cdot 2 \neq 0$ and $\sim-a+a-$ $\sim a=0$. Then $(a \cdot 2) \tau=a \tau \cdot 2$.

Proof. By Lemma 3.1(3) with $b=-a$ we get

$$
(a \cdot 2) \tau-(\sim a-\sim-a) \tau=a \tau .
$$

Now

so we get

$$
\sim a-\sim-a=-(\sim-a-\sim a)=-(-a)=a,
$$

$$
(a \cdot 2) \tau-a \tau=a \tau .
$$

Lemma 10.2. Let $a \in U^{*}$ such that $a \cdot 3=0$ and such that $\sim-a+a-\sim a=0$. Then $\sim a=-a$.

Proof. If $V_{a} \subseteq\{0, a,-a\}$, then by Lemma2.4 $\sim a=-a$. Hence we may assume that $V_{a} \nsubseteq\{0, a,-a\}$. Then, by Corollary 9.5, $\mu_{a}^{2}=1$. By Lemma 10.1, $(a \cdot 2) \tau=(a \tau) \cdot 2$. Similarly $(-a \cdot 2) \tau=(-a) \tau \cdot 2$. We thus have

$$
a \tau=(-a \cdot 2) \tau=(-a) \tau \cdot 2=(a \cdot 2) \tau \cdot 2=a \tau \cdot 4 .
$$

Hence $a \tau \cdot 3=0$. But now $(-a) \tau=(a \cdot 2) \tau=(a \tau) \cdot 2=-(a \tau)$. Hence $(-(a \tau)) \tau^{-1}=$ $-a$, so $\sim a=-a$.

Corollary 10.3. Assume that $U$ is 2-torsion free and let $a \in U^{*}$ such that $V_{a} \nsubseteq \subseteq$ $\{0, a,-a\}$. Then $a \cdot 3=0$ if and only if $\sim a=-a$. 
Proof. First, by Corollary 9.5(2), $\mu_{a}^{2}=1$ and $V_{a}$ is a subgroup of $U$. Let $t=$ $\sim-a+a-\sim a$. By Corollary 9.5 (4), $t \cdot 2=0$, so since we are assuming that $U$ is 2-torsion free, $t=0$.

Assume first that $\sim a=-a$. Then $0=t=\sim-a+a-\sim a=a \cdot 3$. Conversely, assume that $a \cdot 3=0$. Then, by Lemma 10.2, $\sim a=-a$.

Lemma 10.4. Let $a \in U^{*}$ such that $\sim a \neq-a$. Let $t_{a}=\sim-a+a-\sim a$. Then

(1) either $t_{a}$ is the unique involution of $V_{a}$, or $t_{a}=0$ and $V_{a}$ has no involutions;

(2) if $t_{a}=0$, or $t_{a} \neq 0$ and $\sim t_{a}=t_{a}$, then $\sim b \neq b$ for all $b \in V_{a}^{*} \backslash\left\{t_{a}\right\}$, and $a \cdot 2 \neq 0$

(3) there exists $b \in V_{a}^{*}$ such that $b \cdot 2 \neq 0$.

Proof. By Lemma 2.4, $V_{a} \nsubseteq\{0, a,-a\}$, so by Corollary 9.5, $\mu_{a}^{2}=1$ and $V_{a}$ is a root subgroup of $U$.

(1): Let $t \in V_{a}^{*}$ such that $t \cdot 2=0$. By Corollary 9.5(4), $t=\sim-t+t-\sim t=t_{a}$.

(2): Let $x \in V_{a}^{*}$ such that $\sim x=x$. Suppose first that $t_{a}=0$. Then $x \mu_{a}=$ $(\sim x) \mu_{a}=-\left(x \mu_{a}\right)$. Thus $t:=x \mu_{a} \in V_{a}^{*}$ satisfies $t \cdot 2=0$. This contradicts (1). Assume next that $t_{a} \neq 0$ and that $\sim t_{a}=t_{a}$. Then $\sim-x=\sim-x+x-\sim x=t_{a}$. Hence $x=-\sim t_{a}=t_{a}$. Finally if $a \cdot 2=0$, then, by (1), $a=t_{a} \neq 0$, and then $\sim a=-a$, a contradiction.

(3): Assume that $b \cdot 2=0$, for all $b \in V_{a}$. Then, by (1), $V_{a}=\left\{0, t_{a}\right\}$. But since $\sim a,-a \in V_{a}$ we get that $\sim a=-a$, a contradiction.

Lemma 10.5. Let $b \in U^{*}$ such that $V_{b} \nsubseteq\{0, b,-b\}$. Then there exists $a \in V_{b}^{*}$ such that $a \neq \sim a \neq-a$ and $\sim-a \neq-a$.

Proof. Let $t=t_{b}$ as in Corollary 9.5(4). Suppose first that $t \neq 0$ and that $\sim t \neq t$. Then we can take $a=t$.

Assume next that $t=0$ or that $t \neq 0$ and $\sim t=t$. Let $a \in V_{b}^{*}$ such that $\sim a \neq-a$ (see Corollary [.5(1)). Then $a \neq t$, so by Lemma 10.4(2), $a$ satisfies the required properties.

\section{The Proof of the Main Theorem}

In this section we prove the Main Theorem (Theorem 11.6). We assume that $\mathbb{M}(U, \tau)$ is not special, and we show that $\mathbb{M}(U, \tau)$ is sharply 2-transitive.

We denote

$$
\mathbf{N S}:=\left\{a \in U^{*} \mid V_{a} \nsubseteq\{0, a,-a\}\right\} \quad \text { and } \quad \mathbf{S}:=U^{*} \backslash \mathbf{N S} .
$$

Thus $\mathbf{S}$ stands for 'special' (this happens in a special Moufang set) and NS stands for 'not special'. We also let

$$
I:=\left\{a \in U^{*} \mid \mu_{a}^{2}=1\right\} .
$$

Notice that by Corollary 9.5(2),

$$
\mathrm{NS} \subseteq I .
$$

Lemma 11.1. Let $a, b \in U^{*}$ such that $\mu_{a}=\mu_{b}$. Then

(1) if $a \in \mathbf{S}$, then $\sim a=-a$ and $a= \pm b$;

(2) if $\sim a \neq-a$, then $a \in \mathbf{N S}$;

(3) if $a \in \mathbf{N S}$, then $a \tau \in \mathbf{N S}$;

(4) if $a \notin I$, then $a=b$. 
Proof. (1): Since $a \in S$, we have $V_{a} \subseteq\{0, a,-a\}$, so by Lemma 2.4, $\sim a=-a$.

(2): This follows from Lemma 2.4 .

(3): Let $a \in$ NS. It is easy to check that $V_{a \tau}^{*}=V_{a}^{*} \tau$. By Corollary 9.5(1), there exists $c \in V_{a}$ with $\sim c \neq-c$. It follows that $\sim(c \tau) \neq-(c \tau)$. Since $c \tau \in V_{a \tau}$, Lemma 4.6 implies that $a \tau \in \mathbf{N S}$.

(4): Since $a \notin I$ we have $a \in \mathbf{S}$, so by (1), $a= \pm b$.

Now $a \neq-b$; otherwise $\mu_{a}=\mu_{b}=\mu_{-a}$, contradicting $a \notin I$, so $a=b$.

Proposition 11.2. Let $a \in$ NS. Then

(1) there exists $b \in V_{a}$ such that $\sim b \neq-b$;

(2) $\mu_{a}^{2}=1$;

(3) $V_{a}$ is a root subgroup of $U$;

(4) $V_{a}^{*} \subseteq$ NS.

Proof. Parts (1), (2) and (3) are Corollary 9.5. Part (4) follows from (1) and Lemma 4.6 .

Proposition 11.3. Let $a, b \in U^{*}$ and assume that $\sim a \neq-a$ and that $a \neq b$. Then

(1) if $a \neq \sim a$, and $b,(a \tau-b \tau) \tau^{-1} \in I$, then $a-b \in \mathbf{N S}$;

(2) if $\sim-a \neq-a, b \in I$ and $\left(a \tau^{-1}-b \tau^{-1}\right) \tau \notin I$, then $\sim a-\sim b \notin I$;

(3) if $\sim-a \neq-a, b \in I$ and $\left(a \tau^{-1}-b \tau^{-1}\right) \tau \notin I$, then $a-b \in \mathbf{N S}$.

Proof. (1): First, by Lemma 7.3, $\mu_{a-b}=\mu_{\sim a-\sim b}$. Suppose $a-b \notin$ NS. Then, by Lemma 11.1(1), $a-b= \pm(\sim a-\sim b)$, so

$$
\text { either } a-\sim a=b-\sim b \text { or } a+\sim a=b+\sim b .
$$

Suppose that $b \in \mathbf{S}$. By Lemma 11.1(1), $\sim b=-b$. By equation (11.1), $b \cdot 2=$ $b-\sim b=a-\sim a \in V_{a}^{*}$. By Proposition 11.2(4), $b \cdot 2 \in$ NS. By Lemma 8.2 $\mu_{b}=\mu_{b \cdot 2}$, so $b \in V_{b \cdot 2}^{*}=V_{a}^{*} \subseteq \mathbf{N S}$, a contradiction.

Thus $b \in \mathbf{N S}$. Using equation (11.1) and the fact that $V_{a}$ and $V_{b}$ are subgroups we get

$$
\mu_{a}=\mu_{a \pm \sim a}=\mu_{b \pm \sim b}=\mu_{b} .
$$

Working in $V_{a}$ we see that $a-b \in V_{a}^{*} \subseteq \mathbf{N S}$, a contradiction.

(2): Suppose that $\sim a-\sim b \in I$. Then

$$
\left(\left(-a \tau^{-1}\right) \tau-\left(-b \tau^{-1}\right) \tau\right) \tau^{-1}=(\sim a-\sim b) \tau^{-1} \in I .
$$

Let $x:=-a \tau^{-1}$ and $y:=-b \tau^{-1}$. Suppose $\sim x=-x$. Then $(-a) \tau^{-1}=\sim\left(a \tau^{-1}\right)=$ $-\left(a \tau^{-1}\right)=(\sim a) \tau^{-1}$, and then $\sim a=-a$, a contradiction. Suppose $\sim x=x$. Then $\sim-\left(a \tau^{-1}\right)=-a \tau^{-1}$, and then

$$
(-\sim a) \tau^{-1}=\sim-\left(a \tau^{-1}\right)=-\left(a \tau^{-1}\right)=(\sim a) \tau^{-1} .
$$

It follows that $-\sim a=\sim a$ and then, using Lemma 2.3.11) (and the fact that $\mu_{a}^{2}=1$ ), we get $a=\sim-\sim a=-\sim-a$, so $\sim-a=-a$, a contradiction. Also $y,(x \tau-y \tau) \tau^{-1} \in I$ and $x \neq y$. We can thus apply (1) to get $x-y \in I$, that is, $b \tau^{-1}-a \tau^{-1} \in I$. But this implies that $\left(a \tau^{-1}-b \tau^{-1}\right) \tau \in I$, a contradiction.

(3): Set $c:=\left(a \tau^{-1}-b \tau^{-1}\right) \tau$. Notice that since $c \notin I$, we have $c \in \mathbf{S}$, so $\sim c=-c$. By equation (1.4) we have that

$$
\left(a \tau^{-1}-b \tau^{-1}\right) \tau=\left((\sim b) \tau^{-1}-(\sim a) \tau^{-1}\right) \tau=(\sim b-\sim a) \mu_{a}+a,
$$

and since $\left(b \tau^{-1}-a \tau^{-1}\right) \tau=\sim c=-c$ we get

$$
\left(a \tau^{-1}-b \tau^{-1}\right) \tau=-\left(b \tau^{-1}-a \tau^{-1}\right) \tau=-\left[(b-a) \mu_{a}+\sim a\right] .
$$


It follows that

$$
(\sim b-\sim a) \mu_{a}+(b-a) \mu_{a}=-(a+\sim a) .
$$

Interchanging $a$ and $b$ in equation (11.2) (notice that this is possible) we get that

$$
(\sim a-\sim b) \mu_{b}+(a-b) \mu_{b}=-(b+\sim b) .
$$

Notice that by $(2), \sim a-\sim b \in \mathbf{S}$. Assume that $a-b \in \mathbf{S}$. Taking the minus of both sides of the equality in equation (11.3) and using Lemma 11.1(1), we get that

$$
(\sim b-\sim a) \mu_{b}+(b-a) \mu_{b}=b+\sim b .
$$

Applying $\mu_{-a}$ to both sides of equation (11.2) we get

$$
\left((\sim b-\sim a) \mu_{a}+(b-a) \mu_{a}\right) \mu_{-a}=(-(a+\sim a)) \mu_{-a} .
$$

Also, since the LHS of equation (11.2) is nonzero, the LHS of equation (11.4) is nonzero (because $\mu_{-a} \mu_{b} \in \operatorname{Aut}(U)$ ), so we can apply $\mu_{-b}$ to both sides of equation (11.4) to get

$$
\left((\sim b-\sim a) \mu_{b}+(b-a) \mu_{b}\right) \mu_{-b}=(b+\sim b) \mu_{-b} .
$$

Note next that since $b+\sim b \neq 0$, Lemma 11.1(2) implies that $b \in \mathbf{N S}$. Since the LHS of equation (11.5) equals the LHS of equation (11.6), the RHS of the equations are equal. But $(-(a+\sim a)) \mu_{-a} \in V_{a}$ and $(b+\sim b) \mu_{-b} \in V_{b}$, so we get that $\mu_{a}=\mu_{b}$, and now we can conclude that $a-b \in V_{a}^{*} \subseteq$ NS. This contradicts the hypothesis that $a-b \in \mathbf{S}$.

As a corollary we get the following crucial result.

Corollary 11.4. Let $a \in U^{*}$ such that $\sim a \neq-a$, and let $b \in I$ such that $a \neq b$. Then

(1) if $\sim a \neq a$ and $\sim-a \neq-a$, then $a-b \in \mathbf{N S}$;

(2) if $U$ is 2-torsion free, then $a-b \in \mathbf{N S}$;

(3) $\mathbf{N S}=I$.

Proof. Part (1) is immediate from Proposition 11.3, (1) and (3). For (2) notice that since in this part we are assuming that $U$ is 2-torsion free, $\sim x \neq x$, for all $x \in U^{*}$, by Lemma 2.1(1). Hence (2) follows from (1).

Since we know that NS $\subseteq I$, to show (3) it suffices to show that $I \subseteq$ NS. By Lemma 10.5 there exists $c \in V_{a}^{*}$ such that $c \neq \sim c \neq-c$ and $\sim-c \neq-c$. Let $b \in I$. If $b=c$, then $b \in \mathbf{N S}$, so assume $b \neq c$. Using (1) twice we see that $c-b \in \mathbf{N S}$ and then also $b=c-(c-b) \in \mathbf{N S}$.

Theorem 11.5. $I \cup\{0\}$ is a sharply 2-transitive root subgroup of $U$.

Proof. Since $a \mu_{x} \in I$, for every $a \in I$ and $x \in U^{*}$, to show that $I \cup\{0\}$ is a root subgroup of $U$, it suffices to show that $I \cup\{0\}$ is a subgroup of $U$. We work with $\mathbf{N S} \cup\{0\}$ (see Corollary 11.4(3)).

Suppose first that there exists an involution $a \in U^{*}$. Notice that $a \in I$ and hence, by Corollary 11.4(3), $a \in \mathbf{N S}$. Let $b \in \mathbf{N S}$; we show that $V_{a}=V_{b}$. Suppose first that $\sim a \neq a$. Pick $c \in V_{b}^{*}$ with $c \cdot 2 \neq 0$ (see Lemma 10.4(3)). By Corollary 11.4(1), $a+c \in \mathbf{N S}$. Then $c \cdot 2=(c+a) \cdot 2 \in V_{c+a}$ and then $V_{c}=V_{c \cdot 2}=V_{c+a}$. Hence $a, c \in V_{c+a}$, so $V_{a}=V_{c+a}=V_{c}=V_{b}$.

We may thus assume that $\sim t=t$, for all involutions $t \in U$. Hence, either $t_{b}=0$ or $t_{b} \neq 0$ and $\sim t_{b}=t_{b}$ (see Corollary 9.5 for the notation $t_{b}$ ). In both cases pick 
any $c \in V_{b}^{*}$ with $\sim c \neq-c$. Then, by Lemma 10.4, (1) and (2), $c \cdot 2 \neq 0$, and by Lemma 10.4(2), $\sim c \neq c$ and $\sim-c \neq-c$. By Corollary 11.4(1), $c+a \in \mathbf{N S}$ and again $c \cdot 2=(c+a) \cdot 2 \in V_{c+a}$. Again we see that $V_{a}=V_{a+c}=V_{c}=V_{b}$.

Hence we may assume that $U$ is 2-torsion free. Assume first that there exists $a \in \mathbf{N S}$ such that $a \cdot 3=0$. We show that $V_{a}=V_{b}$, for all $b \in \mathbf{N S}$. So let $b \in \mathbf{N S}$ and let $c \in V_{b}$ with $\sim c \neq-c$. By Corollary 10.3, $c \cdot 3 \neq 0$. By Corollary 11.4(2), $c+a \in$ NS. Hence $c \cdot 3=(c+a) \cdot 3 \in V_{c+a}$. Thus $V_{c}=V_{c \cdot 3}=V_{c+a}$. It follows that $V_{b}=V_{c}=V_{a}$, and we are done.

Hence we may assume that $a \cdot 3 \neq 0$, for all $a \in \mathbf{N S}$. By Corollary 10.3 we get that $\sim a \neq-a$, for all $a \in \mathbf{N S}$. By Corollary 11.4(2), $a-b \in \mathbf{N S}$, for all $a, b \in \mathbf{N S}$ with $a \neq b$. This completes the proof that $\mathbf{N S} \cup\{0\}$ is a subgroup of $U$.

Since all $\mu$-maps of $\mathbb{M}(I \cup\{0\}, \tau)$ are involutions, Theorem 8.1 shows that it is sharply 2-transitive (because there exists $a \in I$ with $\sim a \neq-a$ ) and completes the proof of the theorem.

Theorem 11.6. $\mathbb{M}(U, \tau)$ is either special or sharply 2-transitive.

Proof. Suppose that $\mathbb{M}(U, \tau)$ is not special. Then there exists $a \in U^{*}$ such that $\sim a \neq-a$, and by Theorem [9.1, $a \in I$, so $I \neq \emptyset$. By Theorem 11.5, $I \cup\{0\}$ is a sharply 2-transitive root subgroup of $U$. Also, if $U^{*}=I$, then $\mathbb{M}(U, \tau)$ is sharply 2 -transitive, and we are done. Let $a \in I$; we claim that

$$
x \mu_{a}=-x, \quad \text { for all } x \in U^{*} \backslash I .
$$

Indeed let $x \in U^{*} \backslash I$. Then $a \mu_{x} \in I$, so since $I \cup\{0\}$ is a sharply 2-transitive root subgroup, $\mu_{a}=\mu_{a \mu_{x}}=\mu_{-a}^{\mu_{x}}=\mu_{a}^{\mu_{x}}$. Hence $\mu_{x}=\mu_{x}^{\mu_{a}}=\mu_{-x \mu_{a}}$. By Lemma 11.1(4), $x \mu_{a}=-x$, so equation (11.7) holds.

Now let $b \in U^{*} \backslash I$. Then $a+b \in U^{*} \backslash I$. Using equation (11.7) and equation (1.4) we get

$$
(-a) \mu_{a}=(b-(a+b)) \mu_{a}=\left((a+b) \mu_{a}-b \mu_{a}\right) \mu_{a}=a \mu_{b}-b,
$$

where we have used the fact that $\sim b=-b$ since $b \notin I$. Hence we get that $b=$ $a \mu_{b}-(-a) \mu_{a}$. But $a \mu_{b},(-a) \mu_{a} \in I$, which by Theorem 11.5 implies that $b \in I$, a contradiction.

\section{ACKNOWLEDGMENT}

I thank Tom De Medts for numerous remarks that improved the exposition of this paper, and for a very thorough reading of the paper.

\section{ADDED AFTER POSTING}

One additional case should be excluded from Conjecture 2 of the Introduction, the case of $\operatorname{PSU}_{3}(2)(|V|=8)$. Recall that $\operatorname{PSU}_{3}(2) \cong 3^{2}: Q_{8}$ is an elementary abelian group of order 9 extended by the quaternion group $Q_{8}$. I thank Matthias Grueninger from the Free University of Berlin for pointing out this omission to me.

\section{REFERENCES}

[DS1] T. De Medts and Y. Segev, Identities in Moufang sets, Trans. Amer. Math. Soc, 360 (2008), 5831-5852. MR2425693

[DS2] T. De Medts and Y. Segev, Finite special Moufang sets of even characteristic, Commun. Contemp. Math. 10 (2008), no. 3, 449-454. MR2417925

[DS3] T. De Medts and Y. Segev, A course on Moufang sets, Innov. Incidence Geom., to appear. 
[DST] T. De Medts, Y. Segev, and K. Tent, Special Moufang sets, their root groups and their $\mu$-maps, Proc. London Math. Soc. (3) 96 (2008), no. 3, 767-791. MR2407819

[DT] T. De Medts and K. Tent, Special Moufang sets of finite Morley rank, J. Group Theory 11 (2008), no. 5, 645-655. MR2446146

[DW] T. De Medts and R. M. Weiss, Moufang sets and Jordan division algebras, Math. Ann. 335 (2006), no. 2, 415-432. MR2221120 (2007e:17027)

[HKSe] C. Hering, W. M. Kantor and G. M. Seitz, Finite groups with a split BN-pair of rank 1, I, J. Algebra 20 (1972), 435-475. MR0301085 (46:243)

[MV] B. Mühlherr and H. Van Maldeghem, Moufang sets from groups of mixed type, J. Algebra 300 (2006), no. 2, 820-833. MR.2228223 (2007k:20061)

[S1] Y. Segev, Finite special Moufang sets of odd characteristic, Commun. Contemp. Math. 10 (2008), no. 3, 455-475. MR2417926

[S2] Y. Segev, Toward the abelian root groups conjecture for special Moufang sets, submitted.

[SW] Y. Segev and R. M. Weiss, On the action of the Hua subgroups in special Moufang sets, Math. Proc. Cambridge Philos. Soc. 144 (2008), no. 1, 77-84. MR2388234

[Ti1] F. Timmesfeld, Abstract root subgroups and simple groups of Lie type, Birkhäuser-Verlag, Monographs in Mathematics 95 Basel, Berlin, Boston, 2001. MR 1852057 (2002f:20070)

[Ti2] F. Timmesfeld, Quadratic rank-one groups and quadratic Jordan division algebras, Proc. London Math. Soc. (3) 95 (2007), no. 1, 156-178. MR2329550 (2008c:20059)

[T1] J. Tits, Twin buildings and groups of Kac-Moody type, in Groups, combinatorics \& geometry (Durham, 1990), 249-286, London Math. Soc. Lecture Note Ser. 165, Cambridge Univ. Press, Cambridge, 1992. MR1200265 (94d:20030)

[T2] J. Tits, Résumé de cours, Annuaire du Collège de France, 100e année, 1999-2000, 93-109.

Department of Mathematics, Ben-Gurion University, Beer-Sheva 84105, Israel

E-mail address: yoavs@math.bgu.ac.il 